SESSION 7 .

LUMINOUS STELLAR CONTENT OF GALAXIES-INTEGRATED PROPERTIES-I .

Chairman : R.KUDRITZKI.

1. J.G.HOESSEL: Luminosity Functions in Dwarf Irregulars.

2. J.M.SCALO: Initial Mass Function of Massive Stars in Galaxies: Empirical Evidence.

3. C.WHEELER: Luminous Content of Galaxies: Inferences from Supernovae. 
LUMINOSITY FUNCTIONS IN DWARF IRREGULARS

John G. Hoessel

Space Telescope Science Institute

Observing luminosity functions and color-magnitude diagrams is a first step towards understanding the luminous stellar populations in galaxies. Dwarf irregular galaxies are of special interest for a variety of reasons. They are common at distances where they can be resolved, and generally have low background surface brightnesses which makes photometry of the resolved stars relatively easy. Dwarf irregulars exhibit a fairly wide spread in metal abundance allowing the study of massive star formation and evolution under a variety of conditions. Also, the dynamics of such systems is in general simple, without spiral density waves or other effects which might complicate understanding of the star formation process.

The closest and best studied dwarf galaxies are of course the Magellanic clouds, where the work has progressed to detailed investigation of clusters, associations and individual stellar objects. After the clouds the next best known objects are the local group irregulars NGC 6822 and IC 1613. Data on these galaxies is less comprehensive, although several studies of individual clusters and luminous stars have been done. This work is described elsewhere in this volume. The present review will concentrate on observations of less well known systems. The galaxies discussed below in no way constitute a proper statistical sample. Data are taken from the literature where they exist and include unpublished results available to the author. There are certainly strong selections applied, including an emphasis on galaxies which have very low surface brightness.

\section{AVAILABLE DATA}

Photometry exists for the brightest stars in at least 13 galaxies nearby enough to be resolved. Before summarizing the published data, new CCD photometry in the form of color-magnitude (CM) diagrams in the Thuan-Gunn (1976) photometric system will be described. These are preliminary reductions of data which will be discussed in detail elsewhere. In Figure 1 the $G, G-R, C M$ diagram is shown for 3475 measured stars from a mosaic of $9 \mathrm{CCD}$ frames covering the field of NGC 6822. These data will be described in detail by Hoessel and Anderson 


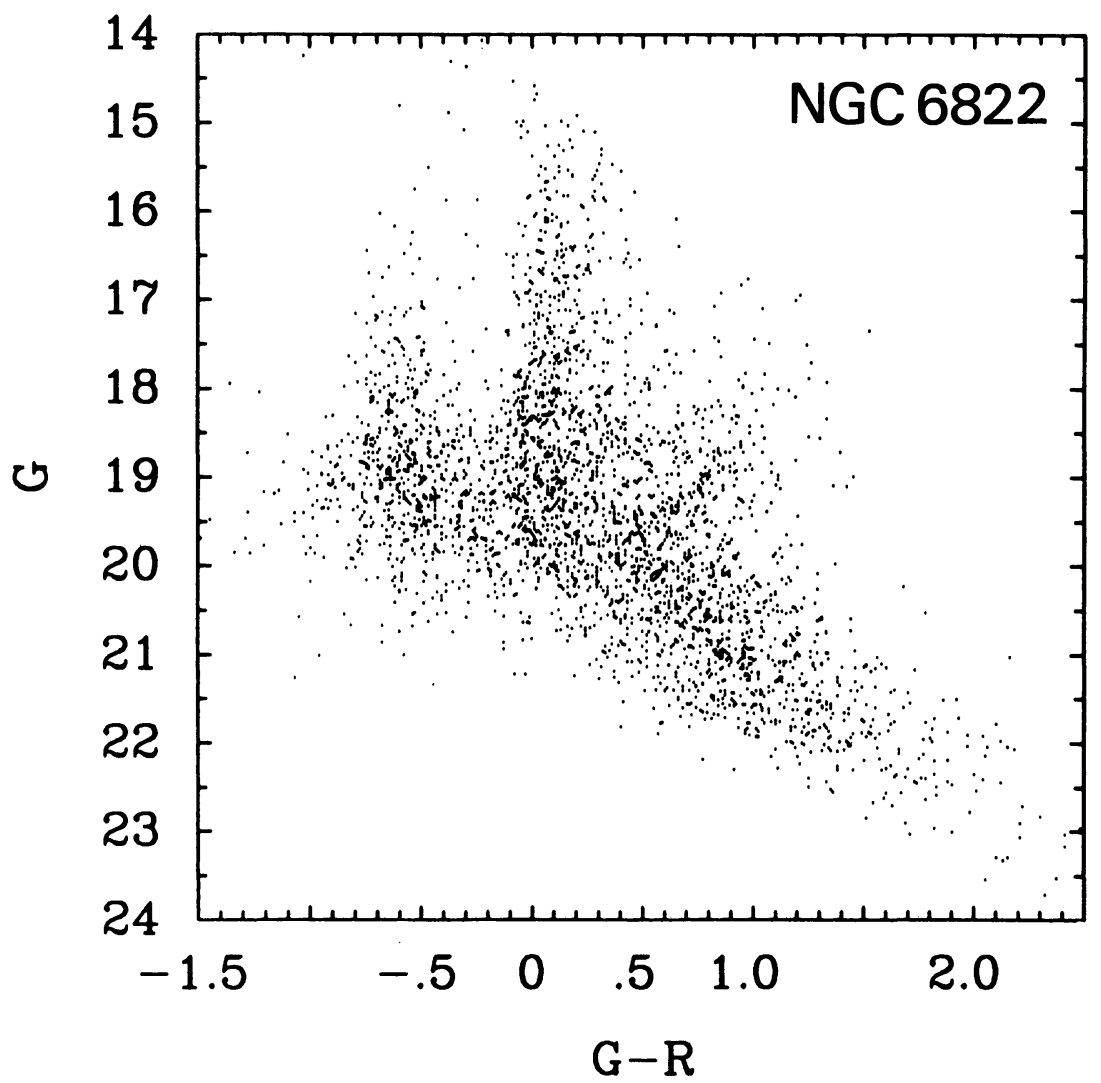

Figure 1--The color-magnitude diagram for NGC 6822. Nine CCD fields were observed on the Thuan-Gunn photometric system. Data are shown for 3475 stellar objects reduced with the aid of RICHFLD.

(1985) along with comparisons star by star with earlier photometry by Kayser (1966) and van den Bergh and Humphreys (1979). Notice in addition to the normal red and blue supergiant branches, the very large number of stars populating the region normally occupied by the Hertzsprung gap. NGC 6822 is located at relatively low galactic latitude. Kayser (1966) showed these to be primarily foreground Galactic stars which complicate any analysis of the luminosity function. However, as discussed by Humphreys and Sandage (1980) if we restrict our attention only to the blue supergiants the foreground contamination over the magnitude range of interest is minimal. This is confirmed for background areas near NGC 6822 by Hoessel and Anderson (1985). In the discussion below only stars with $B-V<0.5$ (i.e. $G-R<0.0$ ) will be considered. These data were reduced using the RICHFLD point spread function fitting software at the KPNO IPPS. The data have been corrected for Galactic reddening assuming $A_{v}=0.90$ mag. 
In Figure 2 new $C C D$ photometry is shown for two galaxies which have published CM diagrams, Sextans $B$ and Leo $A$. Sandage and Carlson (1985a) produced a BV CM diagram for Sextans B, and Leo A was studied by Sandage and Tammann (1982), Demers et. al. (1984), and with new CCD data by Freedman (1984). The photographic data was calibrated with photoelectric sequences. Detailed star by star comparison of the new data by Hoessel and Danielson shown in Figure 2 with the historical record will be done in a later contribution. In Figure 3 new $C C D$ photometry is shown for two galaxies previously unstudied. Based on its kinematics DDO 187 was shown to be nearby by Yahil et. al. (1977), perhaps a companion to G.R.8 (that is number 8 in the list of Reaves, 1956), which Hoessel and Danielson (1983) place near the outskirts of the local group. Here we will adopt a considerably larger distance from the brightest supergiant stars. A photograph of DDO 187 was published by Fisher and Tully (1979). According to Fisher and Tully (1975) DDO 168 is a kinematic member of the Canes venatici I cloud. Note the absence of any observed red supergiants in this galaxy. The photometry of these four galaxies was done with the aid of the DAOPHOT point spread function fitting software developed by P. Stetson.
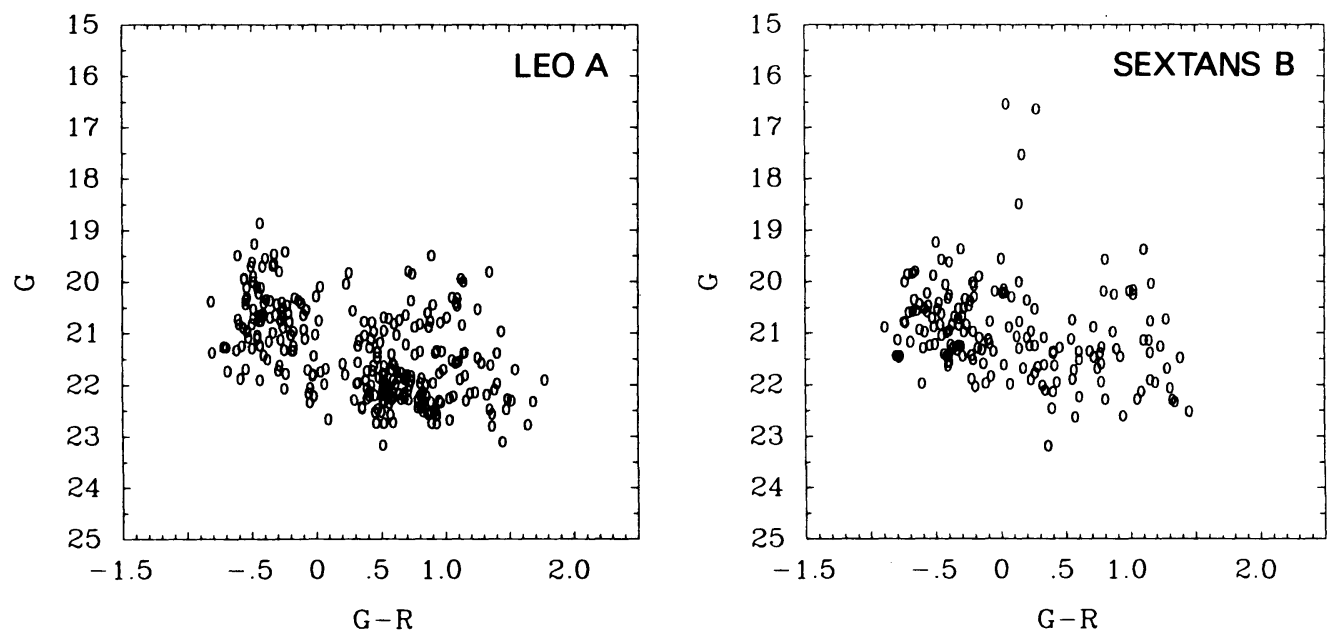

Figure 2--The color-magnitude diagram for Sextans B (DDO 70) and Leo A (DDO 69) from CCD observations reduced with DAOPHOT.

CCD photometry of luminous resolved stars is available in the literature for 7 other nearby dwarf irregulars. Pegasus was studied by Hoessel and Mould (1982). This galaxy also has a photographic CM diagram in Sandage and Tammann (1982). Hoessel and Danielson (1983) list photometry for G.R.8 which has also been surveyed by Hodge (1967). Photometry for stars in Holmberg I and Holmberg II was published by Hoessel and Danielson (1984) and for Sextans $A$ by Hoessel, Schommer and Danielson (1983). Sextans A was studied photographically by Sandage and Carlson (1982) and with a CCD by Freedman (1984). A CCD CM diagram for Holmberg IX has been produced by Freedman (1984). 

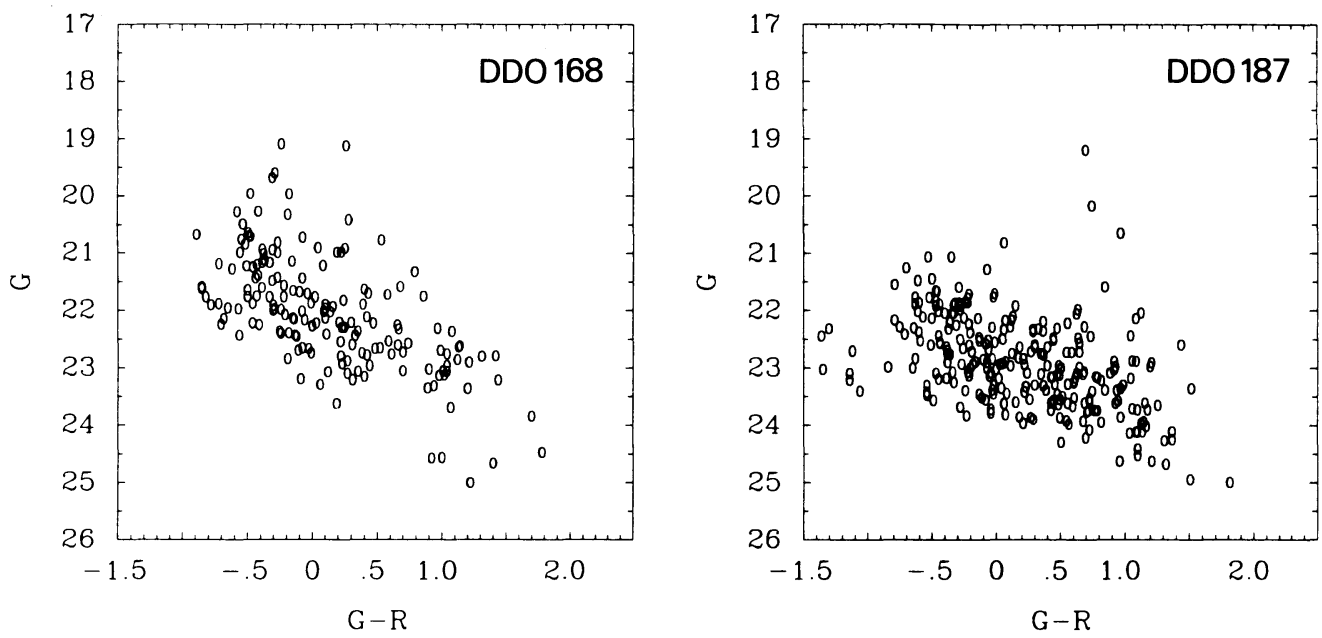

Figure 3--The color-magnitude diagram for DDO 168 and DDO 187 from CCD observations reduced with DAOPHOT. Note the absence of red supergiants in DDO 168.

Finally, two galaxies are included in the present study which as yet have only photographic photometry available for constructing luminosity functions. Sandage and Katem (1976) published a luminosity function for IC 1613. Recently Sandage and Carlson (1985b) have also produced photographic data for WLM. New CCD data have been obtained for both of these galaxies, but are not yet reduced.

Christian and Tully (1983) have shown that the local group irregular LGS-3 has no stars bright enough to be included in the present discussion. This may also be true for other very small stellar systems such as DDO 210, Phoenix, Sagittarius, Sculptor and UKS 2323-326. More detailed observations for each of these systems are necessary.

\section{LUMINOSITY FUNCTIONS}

In order to construct luminosity functions which can be directly compared to one another, two operations must be performed. First, the galactic foreground reddening must be removed. The data described above and shown below were all corrected via the Burstein-Heiles (1978) $21 \mathrm{~cm}$ method. Next a distance modulus for the galaxy is needed to set the absolute magnitude scale. Relatively accurate distances derived from Cepheid light curves exist for only five of the dwarf galaxies in the present sample. These are NGC 6822 (Kayser,1966, McAlary, et al 1983 ), IC 1613 (Sandage 1971, McAlary et al 1984), Sextans A and Sextans B (Sandage and Carlson 1985a), and WLM (Sandage and Carlson 1985b). A common distance of $3.2 \mathrm{Mpc}$ is adopted for the 
three Holmberg galaxies which are in the NGC 2403-M81 group (Tammann and Sandage 1968, Madore 1976, McAlary and Madore 1984). For the remaining galaxies distance estimates from the literature were adopted except for DDO 187 and DDO 168, where the brightest red and blue star calibrations from Sandage and Carlson (1985b) have been adopted. Since DDO 168 has no observed red supergiants only the blue stars were used. Thus, for a majority of the nearby dwarf galaxies accurate distances are not available. This is the major source of uncertainty in interpreting the stellar content in these objects. Many of these galaxies are included in an ongoing program to find Cepheids. The adopted distance modulus for each galaxy is listed in Table 1.

Main sequence (that is, all stars with $B-V<0.5$ ) differential luminosity functions are plotted in Figure 4 for the irregular galaxies discussed above except for Pegasus and G.R.8, where there are too few bright stars to justify a plot. The log number of stars per half magnitude bin is plotted as a function of absolute blue magnitude for the bin for each galaxy, with the number zeropoint shifted down one unit between each data set. Typical error bars, which are based only on the number of stars per bin are attached to two points on the NGC 6822 plot. The galaxies are roughly ordered from top to bottom in decreasing luminous blue star content. The effects of crowding and incompleteness have not yet been fully determined for these data.

The first result is that these plots look rather similar. The slopes at the bright end of the observed luminosity functions are consistent with being a constant value as found for generally more massive systems by Freedman (1984). The average logarithmic slope for the first three galaxies plotted, Holmberg II, NGC 6822, and sextans $A$, is $d(\log N) / d M_{B}=0.53$, but is not well determined. This value is less steep than that found by Freedman (1984). Both this new value for the luminosity function slope, and that found by Freedman (1984) are too shallow to fit with a purely statistical explanation of the correlation between the luminosity of the brightest blue stars and their parent galaxies (Sandage and Katem, 1976). If we assume continuous star formation this slope can be related to the IMF slope by using a mass-luminosity relation and including bolometric corrections. However, since many authors believe that star formation in these small systems occurs in bursts (Searle and sargent, 1972, Gerola et al, 1980), interpretation of the bright-end slope is further complicated by the massive star lifetimes and the detailed chronology in each galaxy. Inspection of Figure 4 showns that smaller, less active systems also have somewhat steeper bright-end slopes as expected. 


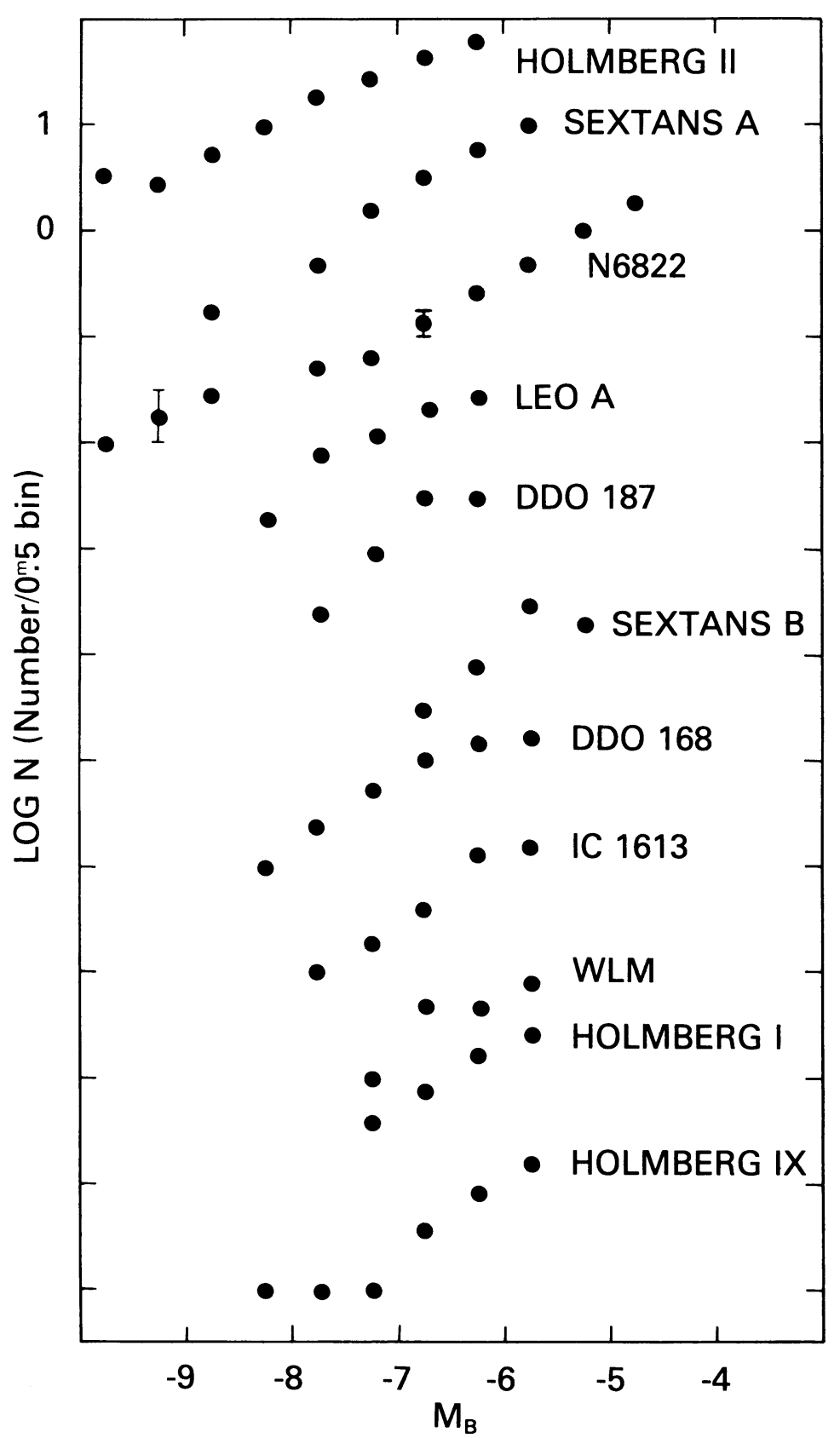

Figure 4-Luminosity functions for all stars with B-V $<0.5$ for 11 dwarf irregular galaxies. The data have been corrected for foreground Galactic extinction and the absolute luminosities are based on the distances listed in Table 1. The log number of stars per half magnitude bin is plotted and the zeropoint of this scale is shifted one unit between each galaxy. 
TABLE 1

Integrated Properties of Dwarf Irregular Galaxies

\begin{tabular}{lllcccc}
\hline Name & Alias & $\mathrm{m}-\mathrm{M}$ & $\mathrm{M}_{\mathrm{H}} / \mathrm{L}$ & $\Delta \mathrm{V}$ & $\log \mathrm{M}_{\mathrm{H}}$ & $\mathrm{M}_{\mathrm{v}}$ \\
\hline DDO 8 & IC1613 & 24.5 & -0.35 & 25 & 7.83 & -15.1 \\
DDO 50 & Ho II & 27.5 & -0.07 & 57 & 8.92 & -16.7 \\
DDO 63 & Ho I & 27.5 & 0.12 & 27 & 8.06 & -14.5 \\
DDO 66 & Ho IX & 27.5 & -- & -- & -- & -13.5 \\
DDO 69 & LeO A & 27.1 & 0.12 & 22 & 8.04 & -14.7 \\
DDO 70 & Sex B & 26.2 & -0.20 & 39 & 7.87 & -14.9 \\
DDO 75 & Sex A & 26.2 & 0.03 & 48 & 8.17 & -14.4 \\
DDO 155 & G.R.8 & 25.7 & -0.05 & 30 & 6.56 & -11.3 \\
DDO 168 & & 27.6 & -0.11 & 63 & 8.29 & -15.5 \\
DDO 187 & & 28.8 & -0.18 & 35 & 8.01 & -14.8 \\
DDO 209 & N 6822 & 23.5 & -0.24 & 61 & 8.14 & -15.5 \\
DDO 216 & Pegasus & 25.3 & -0.53 & 24 & 6.88 & -13.3 \\
DDO 221 & WLM & 25.0 & -0.04 & 54 & 7.84 & -13.9 \\
\hline
\end{tabular}

\section{LUMINOUS STARS AND INTEGRATED PROPERTIES}

Searching for correlations between the properties of luminous stars and their parent galaxies could provide new clues concerning the star formation process and the evolution of galaxies. Several integrated properties of these dwarf irregular galaxies are listed in Table 1. The absolute magnitudes were obtained from the integrated photometry of de vaucouleurs et al (1981) and using the listed distance modulus. The hydrogen mass to luminosity ratios were taken from Fisher and Tully (1975). The $21 \mathrm{~cm}$ widths are full width at $50 \%$ intensity (FWHM) and are from Huchtmeier and Richter (1985), as are the total hydrogen masses. Huchtmeier and Richter (1985) supply the line integrals which have been converted to total masses following the precepts in Fisher and Tully (1975) using distances listed here. In order to attempt to quantify the star formation activity in these galaxies we define a quantity $\mathrm{N}^{\star}$ which is simply the total number of blue $(B-V<0.5)$ stars in the galaxy with $M_{B}$ brighter than -6.0 . In Figure 5 the log of the number of bright blue stars is plotted against the various integrated hydrogen properties of the objects. In the lower panel is the hydrogen mass to optical luminosity ratio which is a distance independent quantity. The central panel shows the bright star numbers in relation to the FWHM of the profile. In some of the larger objects this width indicates systematic rotation in the gas while in some of the smaller galaxies no systematic motion is observed indicating that the gas is turbulent. The upper panel shows the total neutral hydrogen mass as a function of massive star content. Holmberg IX is not included in these figures because its $21 \mathrm{~cm}$ data is badly confused with $M$ 81. The filled circles indicate the existence of a Cepheid distance. The upper panels indicate a trend of increasing 


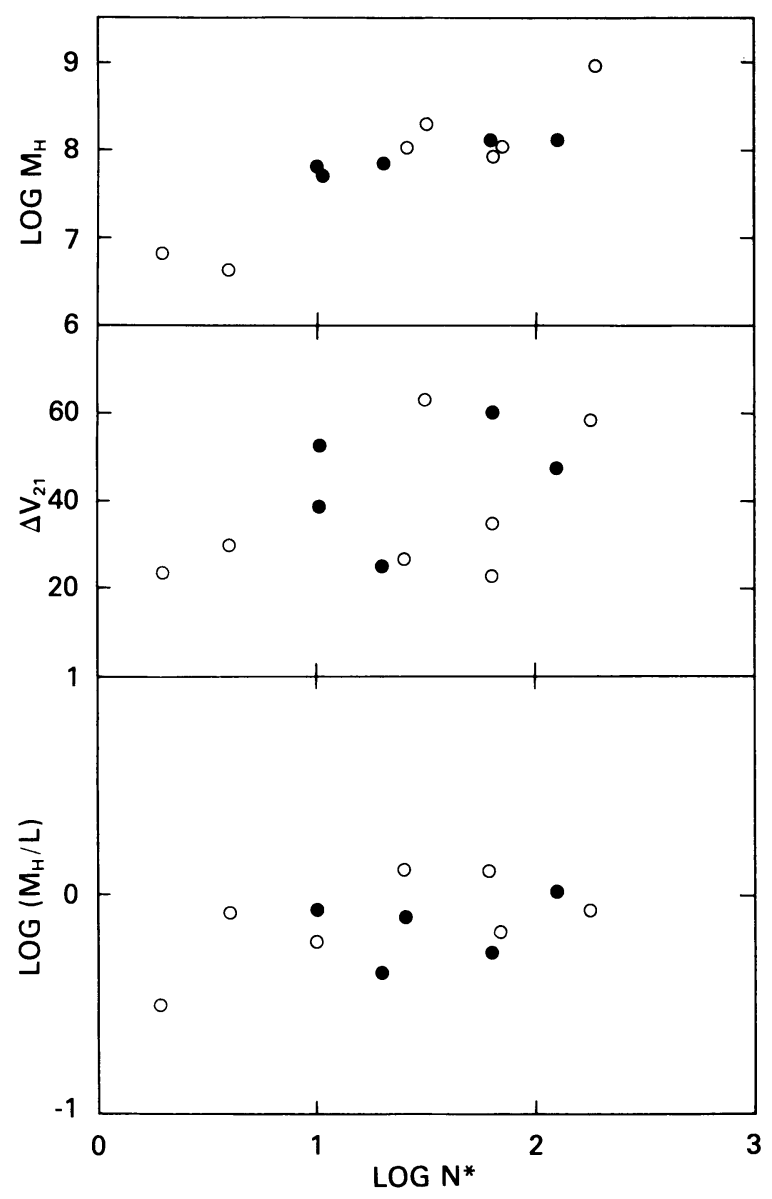

Figure 5--Relations between the number of bright blue stars (i.e. with $M_{B}<-6.0$ and $\left.B-V<0.5\right)$ and the integrated neutral hydrogen properties are shown. Filled circles indicate that a Cepheid light curve distance is available. The lower panel contains the total hydrogen mass to luminosity ratio for the galaxy. The center panel shows the $21-\mathrm{cm}$. 508 intensity full width and the upper panel shows the total neutral hydrogen mass. These parameters are listed in Table 1. Only the total hydrogen mass is distance dependent.

number of bright stars with both larger galaxy dynamical and hydrogen mass. It should be noted that the veracity of these relations depends critically on galaxies with presently uncertain distances, particularly Pegasus and G.R.8. In the lower figure any trend of bright star population with the hydrogen to optical fraction is unclear, especially if the pegasus point is ignored. Improved 
distances are necessary before any conclusions can safely be drawn on the relationship between luminous star content and galaxy hydrogen content.

The relationship between the number of bright blue stars and the total integrated visual magnitude for these galaxies is plotted in Figure 6. Although a trend exists in the sense that more luminous galaxies contain a larger number of massive stars, there is considerable scatter in the data. The luminous stars themselves contribute in general less than 5 percent (eg. Hoessel and Danielson, 1984) of the total visual luminosity of the galaxy. The scatter amounts to as much as a factor of ten in star number at a given galaxy

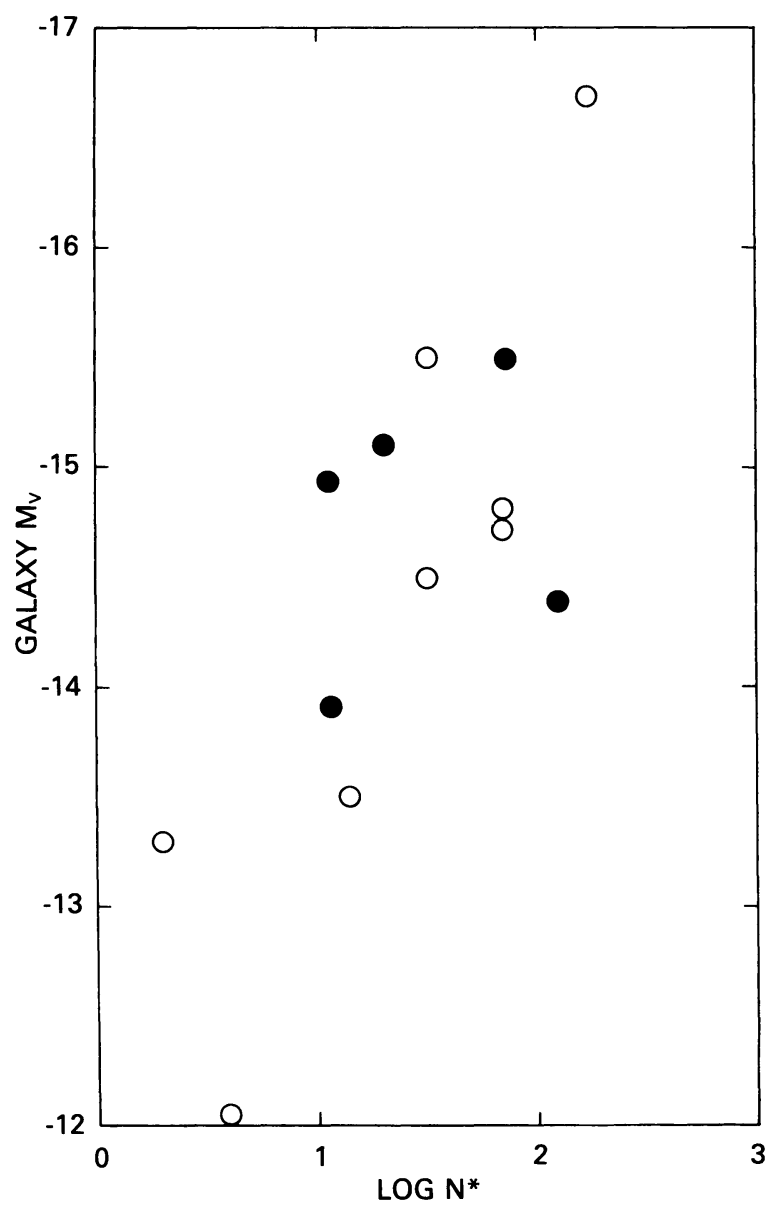

Figure 6--The relationship between the total visual absolute luminosity of the parent galaxy and the number of luminous stars is shown. Filled symbols indicate the existence of a cepheid light curve distance. 
absolute magnitude, or conversely up to 1.5 magnitudes in galaxy luminosity at a given absolute bright star population. There appear therefore to be substantial variations in the ratio of recent star formation activity to past average star formation rate.

\section{FUTURE DIRECTIONS}

In order to improve our understanding of the luminous stellar content in dwarf irregular galaxies new observations will be necessary. A first step is to obtain accurate distances to those galaxies with available photometry. A second step is to include new galaxies which will enlarge the range of integrated properties explored. Possible nearby systems include IC 10 in the northern hemisphere and NGC 3109, IC 5152, DDO 210, Sagittarius, Sculptor and Phoenix in the southern hemisphere. Galaxies in nearby groups such as the M 81 group, Sculptor group, and Canes venatici I cloud will be at the resolution limit with the best ground based observing conditions. The Hubble space Telescope will be a marvelous tool for observations of this.sort. With its superior resolution and faint limiting magnitude the number of objects available for detailed study will be increased by an enormous factor. In a single orbit per color with the wide Field Camera, luminosity functions similar to those shown in Figure 4 can be done at the distance of the virgo cluster. With HST most galaxies in the DDO catalog should be resolved.

Observations of the integrated properties of dwarf irregulars are in relatively good shape, except for their chemical composition which is only known for the very nearest objects. Perhaps the main impact of studies such as those reviewed here will eventually be that they provide finding lists for objects used in a variety of astrophysical studies.

I would like to thank Ed Danielson, Bob Schommer and Nelson caldwell my collaborators on dwarf irregular galaxy projects. Jim Westphal and Jim Gunn have made excellent tools available for this work. I thank Holland Ford for a critical reading of an earlier version of this manuscript.

\section{REFERENCES}

Burstein, D. and Heiles, C.: 1978, Astrophys. J., 225, 40. Christian, C.A and Tully, R.B.: 1983, Astron. J., 88, 934. Demers, S., Kibblewhite, E.J., Irwin, M.J., Bunclark, P.S. and Bridgeland, M.T.: 1984, Astron. J., 89, 1160.

devaucouleurs, G., devaucouleurs, A. and Buta, R.: 1981, Astron. J., 86, 1429 .

Fisher, J.R. and Tully, R.B.: 1975, Astron. Astrophys., 44, 151.

Fisher, J.R. and Tully, R.B.: 1979, Astron. J., 84, 62. 
Freedman, W.L.: 1984, Ph.D. Thesis, University of Toronto.

Gerola, H., Seiden, P.E. and Schulman, L.S.: 1980, Astrophys. J..,

242, 517.

Hodge, P.W.: 1967, Astron. J., 148, 719.

Hoesse 1, J.G. and Anderson, N.: 1985, preprint.

Hoessel, J.G. and Danielson, G.E.: 1983, Astrophys. J., 271, 65.

Hoessel, J.G. and Danielson, G.E.: 1984, Astrophys. J., 286, 159.

Hoessel, J.G. and Mould, J.R.: 1982, Astrophys. J., 254, 38.

Hoessel, J.G., Schommer, R.A. and Danielson, G.E.: 1983, Astrophys. J., 274, 577 .

Huchtmeier, W.K. and Richter, O.G.: 1985, preprint.

Humphreys, R.M. and Sandage, A.: 1980, Astrophys. J. Suppl., 44, 319.

Kayser, S.E.: 1966, Ph.D. Thesis, California Institute of Technology

Madore, B.F.: 1976, M.N.R.A.S., 177, 157.

McAlary, C.W., Madore, B.F., McGonegal, R., McLaren, R.A. and Welch,

D. L.: 1983, Astrophys. J., 273, 539.

McAlary, C.W. and Madore, B.F.: 1984, Astrophys. J., 282, 101.

McAlary, C.W., Madore, B.F. and Davis, L.E.: 1984, Astrophys. J., 276, 487 .

Reaves, G.: 1956, Astron. J., 61, 69.

Sandage, A.: 1971, Astrophys. J.., 166, 13.

Sandage, A. and Carlson G.: 1982, Astrophys. J., 258,439.

Sandage, A. and Carlson, G.: 1985a, preprint.

Sandage, A. and Carlson, G.: 1985b, preprint.

Sandage, A. and Katem, B.: 1976, Astron. J., 81, 743.

Sandage, A. and Tammann G.A.: 1982, in Astrophysical Cosmology, ed. H. A. Bruck, G.V.Coyne, and M.S.Longair (Pontificae Academiae Scientarum Scripta Varia 48), 23.

Searle, L. and Sargent, W.L.W.: 1972, Astrophys. J., 173, 25. Tammann, G.A. and Sandage, A.: 1968, Astrophys. J., $151,825$. Thuan, T.X. and Gunn, J.E.: 1976, P.A.S.P., 88, 543. van den Bergh, S. and Humphreys, R.M.: 1979, Astron. J., 84, 604. Yahil, A., Tammann, G.A. and Sandage, A.: 1977, Astrophys. J., 217, 903.

Discussion. : HOESSEL.

SHARA :

Can you do background subtraction using CCD frames of nearby pieces of sky? What are the problems associated with this technique?

HOESSEL :

This works statistically, but with very few background stars on a given frame. If your interest is specific stars, say red supergiants, then you must look at each one. 